\title{
Factor Analyses of a Hyperiid Amphipod Assemblage from the North Pacific Central Gyre
}

\author{
E. Shulenberger
}

Natural History Museum, P. O. Box 1390, San Diego, California 92112, USA

\begin{abstract}
Abundances of the 20 most common species of hyperiid amphipods in the North Pacific central gyre (28 ${ }^{\circ} \mathrm{N}, 155^{\circ} \mathrm{W}$ ) were studied with factor analysis. Seventy-nine samples were collected with opening/closing nets during a 10-d period: each net tow was about $1000 \mathrm{~m}$ long. Depth intervals were 0-25, 25-50, 50-75, 75-100, 100-350 and 350-600 m. 'R-mode' analyses grouped species with similar patterns of numerical variation across samples. 'Q-mode' analysis grouped samples with similar patterns of variation in species abundances. Orthogonal and oblique factor analyses delineated identical groups of species. Abundances of species within a group vary in parallel ways across samples: abundances within a group are believed correlated with a single underlying factor. Among the 20 species there were about 5 major patterns of variation, each presumably corresponding to an important environmental factor or process. Nineteen species each had a high correlation with one of these factors; the remaining species was consistently highly correlated with more than one factor. The factors are hypothetical constructs derived from the data base, but have yet to be identified with real-world variables. The groups of species were not dominated by patterns of species vertical distributions, diurnal vertical migrations, or relative abundances. The groups of samples were dominated by time of day and depth. The results of factor analyses were compared to an earlier recurrent group analysis of the same data: recurrent group analysis uses only presence/absence. The comparison showed that abundance and presence/absence of species are separable, but not independent, aspects of hyperiid distributional pattern.
\end{abstract}

\section{INTRODUCTION}

Marine zooplankton manifests pattern and interactions on scales ranging from a few millimeters to ocean-wide dimensions. For study of the different scales, different questions are posed and different investigative techniques used. At the small end of the scale is the technique of direct observation of plankton by scuba divers: this is the 'natural history' approach, which elucidates species-species interactions in detail. Such an approach has been most fruitful where zooplankton population densities are relatively high and well represented within the depth zone which limits safe scuba diving (e. g. Hamner et al., 1975; Harbison et al., 1977; Madin and Harbison 1977; Harbison et al., 1978). Such observations are, however, rapidly changing our view of how certain species interact on very fine scales. On ocean-sized scales, patterns of distribution and abundance of organisms, and the correlations of those patterns with environmental features, have been well documented (Ekman 1953; Reid 1962; McGowan 1971, 1974; Reid et al., 1978; see also 'Marine Ecology', Volume V, Part 1).

Between these very large and fine scales are midrange scales on which distributions and abundances of organisms have not been well described. Mid-range scales are probably important ecologically: on scales of a few hundred to a few thousand meters, many pelagic zooplankton species could probably move actively; moreover, the behavior of zooplankters on those scales could significantly affect survival. The methods available to investigate patterns on these scales are at present crude (primarily the use of nets), and suffer major limitations, particularly very slow sample acquisition rates and very long processing times to obtain data from samples. In addition, very little environmental data has been taken simultaneously (i. e. at precisely the same time and place as) with most net samples. In the open sea, there are few abiotic environmental parameters which exhibit strong signals on horizontal scales of a few hundred to a few thousand meters. Hence, it is unlikely that patterns in distributions of organisms on those scales would be explainable in terms of abiotic environmental factors. Instead, biological interactions are most likely to influence patterns of organisms on such horizontal scales. Observations on a scale of a few centimeters to a few meters (e. g. scuba observations on individual organisms) or on scales of hundreds or thousands of kilometers (traditional zoogeographic scales) do not address 
the discovery, description, or explanation of pattern on the mid-range scales. Although new sampling techniques are badly needed for scales of tens to thousands meters, and such techniques are being developed, analyses of available $1000-\mathrm{m}$ scale samples can provide significant preliminary insights into the types and intensities of pattern. The questions asked of $1000-\mathrm{m}$ scale samples may be extremely simple; for example, 'do species covary in abundance across samples?' If they do, then the inference is that some process or force exists which causes those patterns: simple discovery and description of such patterns may not identify the process(es) generating them, but are an important first step. Two essential aspects of pattern are co-occurrence of species and variations in abundances of species. Several recent analyses of zooplankton data have used recurrent group analysis (Fager, 1957) to describe patterns of co-occurrence of species (Fager and McGowan, 1963; Shulenberger, 1976, 1978, 1979; Rottman, 1978; McGowan and Walker, in press). However, recurrent group analysis operates on presence/ absence data and deliberately avoids consideration of variations in, or co-variation of, species abundances, while detecting groups of species which co-occur in samples more frequently than attributable to chance (as discussed by Fager, 1957). After detection of groups, various statistics have been used to investigate crude numerical abundance relationships (e. g. ranks) of group member species (Shulenberger, 1976, 1978, 1979; Mc Gowan and Walker, in press). Missing from these analyses is an attempt to detect, within the original data, patterns based on co-variation of abundances of species with one another. In the present study, factor analysis is used to fill this gap, and to provide another independent description of pattern among these zooplankters. The results are compared with a recurrent group analysis of the same samples (Shulenberger, 1979).

\section{MATERIALS AND METHODS}

All hyperiid amphipods (total $=14,581$ : see Shulenberger, 1977) were sorted, identified, and counted from 79 depth-stratified samples from the North Pacific Central Gyre (at $28^{\circ} \mathrm{N}, 155^{\circ} \mathrm{W}$ : see Fig. 1 in Shulenberger, 1977). All samples were taken with opening/ closing 'BONGO' nets (Scripps Institution of Oceanography, 1966) of $505 \mu \mathrm{m}$ mesh, towed at 3.25 $\mathrm{km} \mathrm{h}^{-1}$; each sample is the zooplankton obtained by one net filtering $400 \mathrm{~m}^{3}$ of water. All were collected during a 10-d sampling period, while maintaining position relative to two parachute drogues deployed at 10 m (see Scripps Institution of Oceanography, 1974, and Shulenberger, 1977, for details of station plan). Depths sampled were $0-25,25-50,50-75,75-100,100-350$, and 350-600 m. Eighty-three hyperiid species were collected.

Factor analysis was used to group hyperiid amphipod species; species within a group should be co-varying numerically (in terms of numbers caught per unit volume of water filtered). Parallel numerical variations are assumed to be functions of some underlying process of force (a factor); there are probably far fewer such fundamental variables than there are original variables in the data. Each original variable is the set of abundances of one species across 79 samples. Factor analysis was also used to group samples. The factors are hypothetical constructs, derived from the data set, which are statements of the existence of such groups of interrelated variables. Several groups of variables may be found within a data set. For example, assume that an analysis includes data on only two types of species: (a) filter-feeding particle harvesters, and (b) obligate visually-feeding carnivores. The abundances of these two groups will tend to vary independently, with the abundances of members within each group covarying: the filter feeders may be most responsive to particle abundance, while the carnivores may depend upon light. Factor analysis would detect these groups whether or not one had any a priori idea that those underlying factors (interrelationships) existed, unless there is a strong correlation between light and particle abundance. If there is such a strong correlation, the groups would likely still be detected, but the factors describing those groups would be correlated with (i. e. not 'orthogonal' to) one another.

The factor analysis program in the Statistical Package for the Social Sciences (Nie et al., 1975) was used. Orthogonal ('VARIMAX') and oblique rotations were performed on a Burroughs 6700 computer using logtransformed data. To determine which species co-vary most closely, I used an 'R-mode' common factor analysis following Rummel (1970, p. 194). The occasion is September 1968; the entities are 79 samples; and the variables are time, depth, and 20 species' numerical abundances in samples. The R-mode analysis delineates groups of variables (i. e. species); members of a group should have similar numerical variations across samples. In addition, to determine which samples are most alike, I used a 'Q-mode' analysis (Rummel, 1970). In the $\mathrm{Q}$-mode analysis the $\mathrm{R}$-mode data matrix was transposed, and the groups are of samples with similar patterns of variation across variables, i. e., across species.

In factor analysis, one assumes that many variables chosen to measure are aspects or realizations of other, more basic underlying processes (termed 'factors') Thus many of the variables measured are correlated 
with one another, either due to cause and effect, or because both are responses to some other underlying process. Factor analysis is a descriptive statistical technique which seeks to discover those underlying relationships and to reproduce (or 'account for') the variation in a data set using as few factors as possible. One assumes an underlying normal multivariate (= multifactor) structure to the universe, and recognizes that the universe can be sampled using any combination of a wide range of possible (correlated) variables. Rummel (1970, p. 104) summarizes factor analysis succinctly: 'the search for common factors is the search for hypothetical variables that will cause the partial correlation between all possible pairs of $m$ variables to tend to zero, when the hypothetical variables are held constant'.

The factor analysis model partitions the total variation in a data set into several categories. 'Communality' is that part of the variance of variable $j$ which is common to the other $n-l$ variables. 'Uniqueness' is that part of $j \mathrm{~s}$ variance not common to the other $n-l$ variables. 'Uniqueness' consists of (1) specific and (2) random error components. Specific variance is always present and is believed to be real (i. e. not measurement error). Random error is noise caused by measurement inaccuracies and the like.

Factor analysis operates upon correlations between measured parameters; it delineates clusters of correlated variables, measures the correlation of the variables with the hypothesized underlying factors, and measures the degree of correlation of those factors with one another. The data set may be visualized as an $n$ dimensional collection of points, where $n=$ number of variables. Factors are new axes derived from the original axes. Because there are fewer factors than original variables, a description of the variation within the data along those factors may be far simpler than a description in terms of the original axes. One hopes to (a) reduce the number of variables significantly, (b) perceive pattern that was obscured by the amount of data, and (c) get significant insights into the causes of pattern in the data. The last depends upon conceptualization of the abstract 'factor' (which is an interrelationship of variables) into a usable and perhaps even directly measureable parameter.

The first step in a factor analysis is calculation of a matrix of correlation coefficients of the $\mathrm{N}$ variables with one another (product-moment correlations were used here). This produces an $N \times N$ matrix in which all diagonal elements (the communalities) are 1.00 . Setting a variable's communality equal to 1.00 assumes that the total variance in the data for that variable is 'explained by' the measured suite of variables (i.e. that one has accurately measured all the variables in the universe which affect the abundances of each species), even though this is certainly an oversimplification.

The second step is to select a more reasonable estimate of communality for each variable. In a sense, this value represent the investigator's view of how well he has selected the variables he measured out of the universe of possible variables: $i$. e., his estimate as to what fraction of the total variation in variable $i$ is 'explained by' (co-)variation of $i$ with all the other measured variables. Two common techniques are to replace the communalities either with the squared multiple correlation coefficient of $i$ with all other variables, or with the maximum correlation coefficient of $i$ with any other variable (see Harman, 1967, and Rummel, 1970, for detailed discussions). The latter is more conservative and computationally economical and is used here.

The third step is an initial extraction of factors, without rotation: moreover orthogonality of factors is enforced (orthogonality means that the factors are uncorrelated). Initially one factor is extracted per original variable. Each successive factor extracts a smaller and smaller percentage of the total variance from the data. The cumulative percentage of total variance extracted is plotted (Fig. 1), and one makes a subjective determination as to how many factors are likely to be meaningful. This is done using established criteria (Harman, 1967; Rummel, 1970). In the present study, the decision to extract 5 factors was based on Figure 1. After specifying five as the number of orthogonal factors to be extracted, the analysis was repeated and all variance was apportioned among them. The resulting set of orthogonal axes could then be rotated until clusters of variables were best delineated ('VARIMAX' rotation was specified: see Harman, 1967; Rummel,

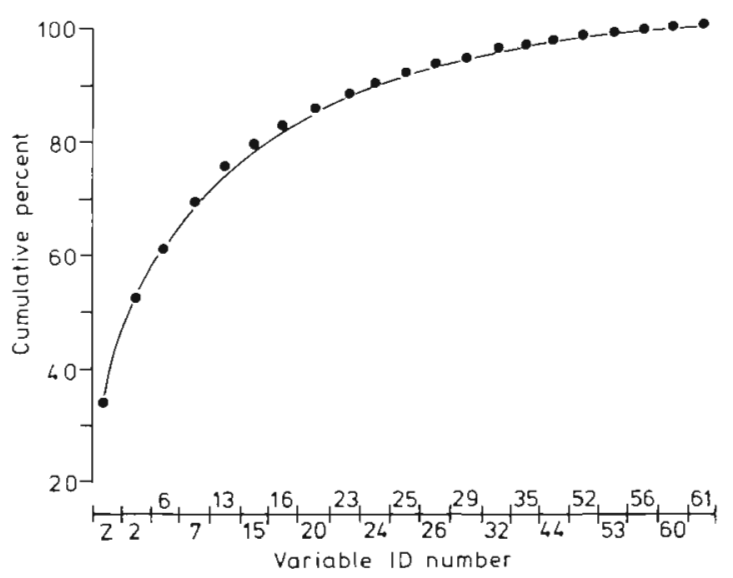

Fig. 1. Cumulative percent of variation extracted from the data set, original unrotated extraction of orthogonal factors. Following criteria in Rummel (1970), five factors were extracted in all subsequent analyses. Numbered variables are species; for names see Table 1 
1970; Nie et al., 1975). This analysis results in an $M \times$ $N$ matrix ( $M=$ \# of factors, $N=$ \# of variables): each element of the matrix is the correlation of an initial variable with a constructed factor. Such values are called factor loadings. The squared loading (correlation) of a variable on a factor equals the fraction of the total variance in that variable, over the entire data set, which is contained in (or 'explained by') that factor. A loading with an absolute value greater than 0.5 (i. e. $\mid$ loading $\mid \geq 0.5$ ) for a variable on a factor means that the factor extracted $\geq 25 \%$ of the total variance in that variable. Such a loading is here called a 'high' loading.

The final step in a factor analysis is to relax the demand that all axes (factors) be orthogonal. This oblique rotation of axes permits: (a) derivation of a more realistic interpretation of the factors that influence the data set, and (b) clearer delineation of the cluster of variables associated with each factor. Oblique rotation seems likely to represent nature more accurately than does orthogonal rotation, because few biological processes in the open ocean are truly independent of one another. In oblique rotation, the previous factor analysis is redone, with new estimates of communality. These are obtained (Rummel, 1970) from the orthogonal analysis by calculating for each variable the sum of the squared loadings (= correlations) of that variable on all factors. For a given species this sum represents the fraction of total variation extracted from the data by the orthogonal analysis, and is the new communality estimate for the species. With the new communalities in the matrix, the factors are computed again without an orthogonality requirement; then the sum of squared loadings is recomputed for each variable, placed in the matrix, and the analysis is repeated until successive iterations converge.

Unfortunately, while making good heuristic sense, oblique rotations generate some problems, especially a lack of uniqueness. Once oblique rotation is specified, one must set up criteria by which to decide when to stop (i. e. decide what constitutes a 'good' oblique rotation). Different criteria (or even different computational methodologies) may give radically different results when operating upon the same original correlation matrix. Discussion of these problems occupies major sections of factor analytic texts (Harman, 1967; Rummel, 1970). The caveat is clear: in all steps beyond calculation of the correlation matrix, factor analytic results are non-unique solutions, and this is particularly true for oblique rotations.

In recurrent group analysis, twenty species were placed into groups (Shulenberger, 1979). Nineteen of the twenty most abundant species were so grouped; they include over $90 \%$ of total individuals in the combined 79 samples. The abundances of those twenty species (ranks \# 1 through 19, plus \#22: names and catches given in Table 1) across all 79 samples are the original data for the factor analyses. The only other variables factored were time of day (scored as $1=$ day, $2=$ night) and depth (scored as 1 through 6 , corresponding to the depth intervals mentioned earlier).

Table 1. Hyperiid amphipod species used in factor analyses. ID \# : identification number used throughout text. ROA: overall rank-order of numerical abundance, 79 samples combined

\begin{tabular}{|c|c|c|c|}
\hline Species & ID \# & $\begin{array}{c}\text { Total } \\
\text { captured }\end{array}$ & ROA \\
\hline Amphithyrus bispinosus & 2 & 70 & 22 \\
\hline Brachyscelus crusculum & 6 & 304 & 11 \\
\hline Anchylomera blossevillei & 7 & 297 & 12 \\
\hline Eupronoe armata & 13 & 336 & 9 \\
\hline Eupronoe sp. A & 15 & 756 & 4 \\
\hline Eupronoe minuta & 16 & 116 & 19 \\
\hline Hyperietta luzoni & 20 & 172 & 16 \\
\hline Hyperietta stephenseni & 23 & 331 & 10 \\
\hline Hyperietta vosseleri & 24 & 3418 & 2 \\
\hline Hyperioides longipes & 25 & 417 & 7 \\
\hline Hyperioides sibaginis & 26 & 956 & 3 \\
\hline Lestrigonus bengalensis & 29 & 632 & 5 \\
\hline Lestrigonus schizogeneios & 32 & 158 & 17 \\
\hline Lycaeopsis themistoides & 35 & 154 & 18 \\
\hline Paratyphis parvus & 44 & 396 & 8 \\
\hline Phronimopsis spinifera & 52 & 252 & 13 \\
\hline Phrosina semilunata & 53 & 225 & 14 \\
\hline Primno latreillei" & 56 & 3695 & 1 \\
\hline Scina crassicornis & 60 & 610 & 6 \\
\hline Scina sp. A. & 61 & 194 & 15 \\
\hline \multicolumn{4}{|c|}{$\begin{array}{l}\text { - Referred to as } P \text {. rectimanus in Shulenberger (1977) } \\
\text { Change follows } T \text {. E. Bowman (pers. comm.). } \\
\text { - } 79 \text { samples combined. }\end{array}$} \\
\hline
\end{tabular}

\section{RESULTS AND DISCUSSION}

\section{Factor Analysis}

The following R-mode factor analyses were performed: \#1, all samples, all variables except time; \#2, all samples, all variables; \#3, all samples, all variables except time and depth; \#4, day samples only, all variables except time; \#5, night samples only, all variables except time. Analysis \#1 will be presented and discussed in greatest detail for three reasons. First, it closely approximates the recurrent group analysis with which factor results will be compared. Second, the oblique results (discussed for \#1 only) did not differ substantially from the VARIMAX (orthogonal rotation) results. Third, the groups of species with high loadings on a particular factor changed little from analysis to analysis. Time was deleted from Analysis \#1 because, as a dichotomy, this variable is quite 
Table 2. Factor analysis results. Each element of each matrix is the I loading I of a variable upon a factor. In VARIMAX, the

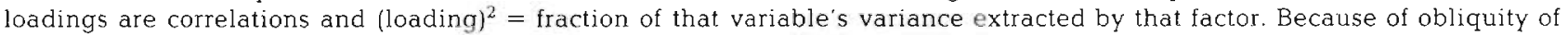
axes, oblique loadings are not strictly correlations (see text). $h^{2}=$ communality = sum of squared VARIMAX loadings for that variable upon all factors: $h_{1}^{2} \times 100=\%$ of total variation in data for variable $i$ extracted by the analysis. VARIMAX extracted $74 \%$ of the total variation in the data set using only 5 factors. All loadings $\geq 0.5$ are enclosed in parentheses and are those loadings used to define groups. $Z=$ depth

\begin{tabular}{|c|c|c|c|c|c|c|c|c|c|c|c|c|c|c|c|c|}
\hline \multirow[b]{2}{*}{ Variable } & \multicolumn{6}{|c|}{ VARIMAX (ORTHOGONAL) } & \multicolumn{5}{|c|}{ OBLIQUE PATTERN } & \multicolumn{5}{|c|}{ OBLIQUE STRUCTURE } \\
\hline & $F_{1}$ & $\mathrm{~F}_{2}$ & $\mathrm{~F}_{3}$ & $\mathrm{~F}_{4}$ & $\mathrm{~F}_{5}$ & $\mathrm{~h}^{2}$ & $F_{1}$ & $F_{2}$ & $\mathrm{~F}_{3}$ & $\mathrm{~F}_{4}$ & $F_{5}$ & $F_{1}$ & $\mathrm{~F}_{2}$ & $\mathrm{~F}_{3}$ & $\mathrm{~F}_{4}$ & $\mathrm{~F}_{5}$ \\
\hline Z & $(.74)$ & .30 & .03 & .34 & .09 & .76 & 18 & .02 & $(.62)$ & 16 & .31 & .43 & .05 & $(.77)$ & .30 & $(.53)$ \\
\hline 2 & .25 & $(.71)$ & .04 & .02 & .07 & .57 & $(.73)$ & .09 & 13 & .06 & .03 & $(.74)$ & 18 & 33 & 21 & 14 \\
\hline 6 & $(.69)$ & .46 & .02 & .08 & .09 & .70 & .40 & .04 & $(.63)$ & .05 & .06 & $(.56)$ & .13 & $(.75)$ & 11 & .29 \\
\hline 7 & $(.79)$ & .30 & .19 & 27 & .22 & .87 & 20 & .20 & (.66) & .30 & .22 & .42 & .09 & (.81) & .43 & $(.50)$ \\
\hline 13 & .31 & .24 & .24 & .34 & 17 & 36 & .12 & .26 & 16 & 19 & .33 & 35 & .29 & .33 & 30 & 38 \\
\hline 15 & .49 & .16 & .39 & .41 & .06 & .59 & .01 & .46 & .35 & .09 & .42 & .33 & .43 & $(.50)$ & .23 & .46 \\
\hline 16 & $(.72)$ & .08 & .04 & 15 & .23 & .60 & .14 & .03 & $(.82)$ & .15 & .15 & .03 & .02 & $(.72)$ & 10 & .04 \\
\hline 20 & .07 & .18 & $(.52)$ & .04 & 18 & .34 & .08 & $(.50)$ & .01 & .16 & .05 & .28 & (.55) & .07 & .22 & .08 \\
\hline 23 & .30 & .44 & .43 & .42 & .20 & .68 & .43 & .30 & .36 & .22 & .44 & $(.52)$ & $(.51)$ & .35 & .11 & .34 \\
\hline 24 & .06 & $(.66)$ & (.63) & .13 & 17 & .88 & $(.57)$ & $(.55)$ & .11 & .12 & .10 & $(.77)$ & $(.74)$ & .13 & .29 & .11 \\
\hline 25 & .18 & .06 & .27 & $(.73)$ & .05 & .64 & .02 & 17 & .01 & .02 & $(.76)$ & 08 & 26 & .21 & .06 & $(.79)$ \\
\hline 26 & .24 & $(.66)$ & 17 & .49 & .08 & .77 & $(.60)$ & 13 & .00 & .08 & .47 & $(.73)$ & .28 & .32 & .27 & $(.56)$ \\
\hline 29 & $(.70)$ & .23 & 11 & .31 & .30 & .74 & .07 & .13 & $(.55)$ & .36 & .27 & .39 & 19 & $(.70)$ & .49 & .47 \\
\hline 32 & .15 & $(.81)$ & .01 & .04 & .10 & .69 & $(.86)$ & 15 & .02 & .07 & 10 & $(.82)$ & .16 & .24 & .22 & .07 \\
\hline 35 & .27 & $(.67)$ & .31 & .13 & .01 & .64 & $(.63)$ & .22 & .14 & .01 & 10 & $(.76)$ & .43 & .36 & .17 & .21 \\
\hline 44 & .13 & $(.84)$ & .12 & .29 & .06 & .83 & $(.85)$ & .01 & .08 & .03 & .26 & $(.87)$ & .27 & .23 & .22 & 38 \\
\hline 52 & .14 & (.53) & .47 & .32 & .03 & .63 & $(.55)$ & .33 & .16 & .04 & .35 & (.56) & (.55) & 18 & .04 & .35 \\
\hline 53 & .04 & .01 & $(.75)$ & 12 & .09 & .59 & .13 & $(.76)$ & .03 & .06 & .12 & 14 & $(.75)$ & .02 & 10 & .22 \\
\hline 56 & .26 & 11 & $(.90)$ & 11 & .26 & .97 & .04 & $(.90)$ & .23 & .34 & .06 & 21 & (88) & .22 & 28 & .28 \\
\hline 60 & .06 & .16 & .07 & 10 & $(.82)$ & 73 & .09 & .01 & .07 & (.84) & .20 & 21 & .14 & .00 & $(.82)$ & 10 \\
\hline 61 & .18 & 12 & 18 & $(.59)$ & .37 & .57 & 11 & 10 & .08 & .35 & $(.65)$ & 13 & .18 & .24 & .25 & $(.66)$ \\
\hline
\end{tabular}

different from the others and could cause problems in interpretation of results.

Table 2 lists the results of analysis \#1. The first step (original unrotated orthogonal factors) is not presented. The rotated orthogonal factors ('VARIMAX') and obliquely rotated factors are shown, along with other information. All 'high' loadings are enclosed in parentheses. In the VARIMAX results, there are several obvious trends. Each factor tends to include a few high loadings, many low loadings, and few intermediates. Most variables do have at least one high loading on some factor (exceptions are \#13,15 and 23). Only one variable (Hyperietta vosseleri) has high loadings on more than one factor (var \#24, on F2 and F3). I define a group as being the set of variables having | loading $\mid \geq 0.5$ on a particular factor.

The species with high loadings on a particular factor all tend to vary numerically in parallel ways across samples. This implies parallel responses to some environmental process. The underlying process (or forcing function) is unlikely to be vectorial (i.e. physical) in nature, since such a process should affect all species more or less equally, and they would therefore all have had high loadings on the same factor. Underlying the total variance in the data there are a few major axes (factors) along which the data are distributed; individual species respond most strongly to (i.e. correlate with) only one of that suite, rather than with several.

Each communality value (Column $\mathrm{h}^{2}$ ) in Table 2 is the sum of the squared loadings of that variable on each of the 5 VARIMAX factors. The 'ith' communality therefore represents the fraction of the total variation for species, which has been accounted for by the

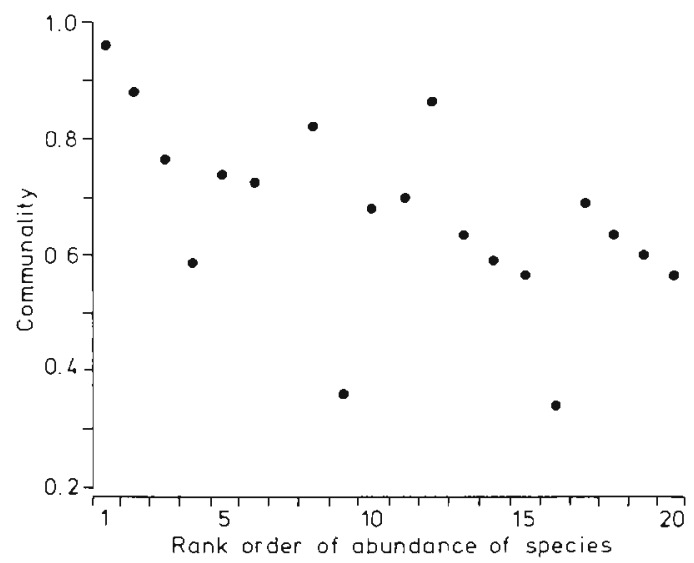

Fig. 2. Rank order of numerical abundance vs communality estimate. Olmstead and Tukey's 'corner test' for association was used to check the null hypothesis that no correlation exists; $\mathrm{H}_{\mathrm{O}}$ rejected but only at $\mathrm{P} \approx 0.10$. Communality: fraction of the total variance in data on variable $e_{i}$ extracted by the analysis 
extraction of those 5 orthogonally-rotated factors. Communalities range from 0.97 (i.e., $97 \%$ of variation extracted; var. \#56) to 0.34 (= $34 \%$; var. \#20). The VARIMAX factor analysis accounted for $74 \%$ of the total variation in the data set (Table 2). Communality values appear to be weakly correlated with rank order of numerical abundance of species (Fig. 2); some such correlation is not unexpected. It appears reasonable to be able to extract more of the variation in data on those species for which the largest catches are available. The correlation is only significant at $P=0.10$; the test used (Olmstead and Tukey's corner test: Sokal and Rohlf, 1969 ) is quite conservative, however. Use of a more distribution-dependent test is probably not warranted.

The oblique 'pattern' and 'structure' matrices (Table 2) strongly resemble one another. However, the use of each is rather different (Harman, 1967; Rummel, 1970). The pattern matrix best displays the group of variables associated with each factor, while the structure matrix approximates a set of correlations of variables with factors. For both oblique matrices, however, the loadings are not simple correlations of variables with factors since the factors are not orthogonal to one another. Loadings on oblique factors do resemble correlations in that a value near zero indicates very little relationship between variable and factor, while values near 1.0 indicate the converse.

Factor numbers are arbitrary; groups of variables are the important part of the results. Here, oblique and VARIMAX groups are identical (Table 3). Only values of loadings have changed slightly (Table 2), as the

Table 3. Groups of variables with high loadings on each factor; all samples, all variables except time. A: VARIMAX orthogonal rotation. B: oblique rotation, pattern matrix. VF:

VARIMAX factor number. OF: oblique factor number

\begin{tabular}{|ll|}
\hline Factor $\begin{array}{c}\text { (A.) VARIMAX } \\
\text { Variable numbers }\end{array}$ & $\begin{array}{c}\text { (B.) OBLIQUE PATTERN } \\
\text { Factor Variable numbers }\end{array}$ \\
\hline VF1 depth, $6,7,16,29$ & OF3 depth, 6, 7, 16, 29 \\
VF2 $2,24,26,32,35,44,52$ & OF1 $2,24,26,32,35,44,52$ \\
VF3 20,24,53,56 & OF2 $20,24,53,56$ \\
VF4 25,61 & OF5 25,61 \\
VF5 60 & OF4 60 \\
\hline
\end{tabular}

Table 4. Correlations between oblique factors, from Analysis 1 (all samples, all variables except time)

\begin{tabular}{cccccc|}
\hline$F_{1}$ & $F_{2}$ & $F_{3}$ & $F_{4}$ & $F_{5}$ & \\
\hline$\times$ & .34 & .28 & .20 & .16 & $F_{1}$ \\
& $\times$ & .06 & 10 & 13 & $F_{2}$ \\
& & $\times$ & 12 & .28 & $F_{3}$ \\
& & & $\times$ & 12 & $F_{4}$ \\
& & & & $\times$ & $F_{5}$ \\
\hline
\end{tabular}

factors are allowed to best delineate groups. The robustness of group membership despite change in analytical technique suggests that the group members are indeed responding similarly to some force or process.

In the oblique analysis, any apparent independence of factors is presumably real. Factor interrelationships (i.e. degee of non-orthogonality) may be measured as correlation coefficients of one factor against another. Table 4 presents those coefficients for analysis (1). The structure of the 5-factor space is relatively simple: there are no highly correlated pairs of factors. Even when rotated to simple structure (i.e. permitted to be non-orthogonal), the factors tend to be independent of one another. Squaring such a correlation coefficient. gives the fraction of a factor's variance which it shares with the other factor. There are no available methods for accurately computing significance levels for correlations between oblique factors. The highest correlation is $F_{1} \times F_{2}$ (coeff. $=0.34$ ); these factors share about $11 \%$ of their variance, and are, to that degree, dependent (non-orthogonal). The $\mathrm{F}_{1} \times \mathrm{F}_{2}$ interrelationship is probably due to Hyperietta vosseleri (\#24) which had high loadings on both oblique $F_{1}$ and $F_{2}$ (Table 2): it is the second most abundant species and is the only variable to have high loadings on more than one factor. The lowest oblique correlation is $\mathrm{F}_{3} \times \mathrm{F}_{2}$ : these factors share essentially no variation $\left(0.06^{2}=0.0036\right)$, i.e., they are almost orthogonal. $F_{1} \times F_{3}$ and $F_{5} \times F_{3}$ both have correlations of 0.28 . Both pairs have several species with medium loadings on both pair members (Table 2).

Whatever the forcing functions are that generate these sets of patterns of variation, they appear to be almost entirely dissociated from (i.e. uncorrelated with) one another. Certainly they are less connected than one would expect in the hypothetical herbivore vs carnivore example given in the introduction, since those two sets may both be strongly controlled by light intensity and therefore should have some correlation with one another.

Table 5 presents the species groupings derived from the five different analyses used to interpret the data. The close similarity between orthogonal and oblique species groups was maintained in the other four analyses, so only the orthogonal results are shown. The three analyses which included all 79 samples (Table $5 \mathrm{a}, \mathrm{b}, \mathrm{c})$ show very similar results.

Shulenberger (1978) showed that, among the 10 most abundant hyperiid species studied here, little could be statistically documented in the way of diurnal vertical migrations or depth preferences in the upper $100 \mathrm{~m}$. The addition or removal of time and depth as factored variables produced no major change in groupings: this substantiates those earlier conclusions. Lestrigonus bengalensis (\#29) was shown (Shulenberger, 1978) to 
Table 5. Results of five VARIMAX factor analyses. Factor numbers are arbitrary. Only high-loaded species are shown (see text). F: factor number; numbers in columns are variable ID\#s (Table 1); Z: depth; T time of day. (A) All 79 samples, all variables except $T$ (see text). (B) All 79 samples, no $T$ or $Z$ (C) All 79 samples, all variables. (D) Night samples only, all variables except $T$ (E) Day samples only, all variables except $T$ Number in parentheses are factor loadings; because these are orthogonal factors, $100 \times$ (loading) $^{2}=$ percent of that variable's variance extracted by the analysis

\begin{tabular}{|c|c|c|c|c|c|c|c|c|c|}
\hline \multicolumn{2}{|r|}{$\mathrm{F}_{1}$} & \multicolumn{2}{|r|}{$\mathrm{F}_{2}$} & \multicolumn{2}{|r|}{$\mathrm{F}_{3}$} & \multicolumn{2}{|r|}{$\mathrm{F}_{4}$} & \multicolumn{2}{|c|}{$\mathrm{F}_{5}$} \\
\hline & & & & & A & & & & \\
\hline Z & $(.74)$ & 2 & $(.71)$ & 20 & $(.52)$ & 25 & $(.73)$ & 601 & $(.82)$ \\
\hline 6 & $(.69)$ & 24 & (.66) & 24 & (.63) & 61 & $(.59)$ & & \\
\hline 7 & $(.79)$ & 26 & $(.66)$ & 53 & $(.75)$ & & & & \\
\hline 16 & $(.72)$ & 32 & $(.81)$ & 56 & $(.90)$ & & & & \\
\hline \multirow[t]{4}{*}{29} & $(.70)$ & 35 & $(67)$ & & & & & & \\
\hline & & 44 & $(.84)$ & & & & & & \\
\hline & & 52 & (.53) & & & & & & \\
\hline & & & & & B & & & & \\
\hline 2 & $(.71)$ & 23 & $(.51)$ & 6 & $(.55)$ & 7 & (.64) & 601 & $(.78)$ \\
\hline 24 & $(.63)$ & 24 & $(.59)$ & 7 & (.51) & 13 & $(.54)$ & 61 & $(.60)$ \\
\hline 26 & $(.65)$ & 52 & $(.54)$ & 16 & $(.74)$ & 15 & $(.67)$ & & \\
\hline 32 & $(.82)$ & 53 & $(.74)$ & & & 25 & $(.57)$ & & \\
\hline 35 & $(.67)$ & 56 & (.95) & & & 26 & $(.56)$ & & \\
\hline 44 & $(.83)$ & & & & & 29 & $(.71)$ & & \\
\hline \multirow[t]{2}{*}{52} & $(.52)$ & & & & & & & & \\
\hline & & & & & C & & & & \\
\hline 20 & $(.53)$ & 25 & $(.72)$ & 2 & $(.70)$ & Z & $(.76)$ & T 1 & $(.66)$ \\
\hline 24 & $(.64)$ & 61 & (.55) & 24 & (.66) & 6 & (.69) & 601 & $(.87)$ \\
\hline 53 & $(.77)$ & & & 26 & $(.66)$ & 7 & (.81) & & \\
\hline \multirow[t]{5}{*}{56} & $(.85)$ & & & 32 & (.81) & 15 & $(.50)$ & & \\
\hline & & & & 35 & $(.68)$ & 16 & $(.70)$ & & \\
\hline & & & & 44 & $(.84)$ & 29 & $(.73)$ & & \\
\hline & & & & & (.54) & & & & \\
\hline & \multicolumn{9}{|c|}{$\mathrm{D}$} \\
\hline Z & $(.80)$ & 7 & $(.51)$ & 20 & $(.64)$ & 23 & $(61)$ & & $x$ \\
\hline 2 & $(.78)$ & 13 & (.51) & 53 & (.85) & 25 & $(.77)$ & & \\
\hline 6 & $(.91)$ & 15 & (.89) & 56 & $(.72)$ & 52 & $(.80)$ & & \\
\hline 7 & $(.78)$ & 24 & $(.56)$ & 60 & $(.60)$ & & & & \\
\hline 24 & $(68)$ & 26 & (.58) & 61 & (.58) & & & & \\
\hline 26 & $(.76)$ & 29 & (.81) & & & & & & \\
\hline 32 & $(83)$ & 60 & $(.56)$ & & & & & & \\
\hline 35 & $(.78)$ & & & & & & & & \\
\hline & $(87)$ & & & & & & & & \\
\hline & & & & & $E$ & & & & \\
\hline 2 & $(.58)$ & z & $(.79)$ & 25 & $(.54)$ & 26 & $(.64)$ & 201 & $(.78)$ \\
\hline 23 & $(.78)$ & 6 & $(.75)$ & 60 & (.61) & & & & \\
\hline 24 & $(86)$ & 7 & $(.88)$ & & & & & & \\
\hline 26 & $(.55)$ & 15 & (.61) & & & & & & \\
\hline 32 & $(.58)$ & 16 & $(.70)$ & & & & & & \\
\hline \multirow{2}{*}{$\begin{array}{l}35 \\
44\end{array}$} & $(.61)$ & 29 & $(.77)$ & & & & & & \\
\hline & (.61) & & & & & & & & \\
\hline 52 & $(.75)$ & & & & & & & & \\
\hline 53 & $(.63)$ & & & & & & & & \\
\hline 56 & (.94) & & & & & & & & \\
\hline
\end{tabular}

have a pronounced peak of abundance at 75-350 m, which probably explains why it usually grouped with depth when depth was used as a variable (Table $5 a, c$, e).

There are pronounced changes in group makeup, however, when day and night samples are analyzed separately (Table 5d, e). Species \#2, 24, 26, 32, 35, and 44 are the core of the $F_{1}$ group and load high on $F_{1}$ both day and night. At night, species \#52,53,56, and 23 split off from this group (with which they are associated during the day). However, species \#53 and 56 remain together, as they did through all other analyses. Variable depths, \#6, and \#7, which were grouped on $F_{2}$ during the day, moved to night $F_{1}$.

Results of the ' $Q$-mode' analysis are presented in Table 6 . In this analysis, samples are grouped by

Table 6. Samples which had high loadings (loading $\geq 0.7$ on $\mathrm{F} 1-\mathrm{F} 3, \geq 0.5$ on F4) on factors in Q-mode analysis. $\mathrm{S} \#$ : sample number; $T$ : time of day $(D==$ day, $N=$ night); $Z$ : depth interval $(1=0-25 \mathrm{~m}, 2=25-50 \mathrm{~m}, 3=50-75 \mathrm{~m}, 4=75-100 \mathrm{~m}$ $5=100-350 \mathrm{~m}, 6=350-600 \mathrm{~m})$

\begin{tabular}{|c|c|c|c|c|c|c|c|c|c|c|c|}
\hline \multicolumn{3}{|c|}{ Factor 1} & \multicolumn{3}{|c|}{ Factor 2} & \multicolumn{3}{|c|}{ Factor 3} & \multicolumn{3}{|c|}{ Factor 4} \\
\hline $\mathrm{S} \#$ & $\mathrm{~T}$ & Z & $\mathrm{S} \#$ & $\mathrm{~T}$ & $Z$ & $S \#$ & $\mathrm{~T}$ & Z & S\# & $\mathrm{T}$ & Z \\
\hline 3 & $\mathrm{D}$ & 3 & 25 & $\mathrm{~N}$ & 1 & 6 & D & 6 & 1 & D & 1 \\
\hline 4 & $\mathrm{D}$ & 4 & 32 & $N$ & 1 & 11 & D & 5 & 66 & D & 1 \\
\hline 9 & $\mathrm{D}$ & 3 & 37 & $N$ & 2 & 44 & $D$ & 6 & 72 & $D$ & 1 \\
\hline 10 & $\mathrm{D}$ & 4 & 46 & $N$ & 1 & 31 & D & 6 & 74 & D & 1 \\
\hline 13 & $\mathrm{D}$ & 2 & 83 & $N$ & 1 & & & & 81 & D & 1 \\
\hline 14 & $\mathrm{D}$ & 2 & 85 & $N$ & 2 & & & & & & \\
\hline 15 & $\mathrm{D}$ & 3 & & & & & & & & & \\
\hline 19 & $\mathrm{D}$ & 4 & & & & & & & & & \\
\hline 20 & $\mathrm{D}$ & 4 & & & & & & & & & \\
\hline 42 & D & 5 & & & & & & & & & \\
\hline 43 & D & 5 & & & & & & & & & \\
\hline 54 & D & 3 & & & & & & & & & \\
\hline 56 & $\mathrm{D}$ & 3 & & & & & & & & & \\
\hline 70 & $\mathrm{D}$ & 4 & & & & & & & & & \\
\hline 71 & $\mathrm{D}$ & 3 & & & & & & & & & \\
\hline 78 & $\mathrm{D}$ & 3 & & & & & & & & & \\
\hline 79 & $\mathrm{D}$ & 4 & & & & & & & & & \\
\hline 82 & $\mathrm{D}$ & 1 & & & & & & & & & \\
\hline 84 & $\mathrm{D}$ & 4 & & & & & & & & & \\
\hline 21 & $N$ & 5 & & & & & & & & & \\
\hline 22 & $N$ & 5 & & & & & & & & & \\
\hline 28 & $N$ & 5 & & & & & & & & & \\
\hline 39 & $N$ & 3 & & & & & & & & & \\
\hline 53 & $N$ & 4 & & & & & & & & & \\
\hline 61 & $\mathrm{~N}$ & 4 & & & & & & & & & \\
\hline 63 & $\mathrm{~N}$ & 4 & & & & & & & & & \\
\hline
\end{tabular}

similarities of abundances of the species found in each sample. The data analyzed were abundances of each of 20 species in each of 79 samples; depth and time of day were not included, permitting a posteriori examination of sample groups to see whether group membership was dominated by those parameters. The analysis extracted 12 (orthogonal) factors, and the first four extracted $38 \%, 22 \%, 11 \%$, and $8 \%$, respectively, of the total variance. There were no $\mid$ loadings $\mid>0.50$ on factors \#5-12. No sample had loadings $\geq 0.5$ on more than one factor.

On factors \#1-3, the groups (Table 6) consist of all samples with $\mid$ loading $\mid \geq 0.70$ on that factor: such a 
loading means that over $(0.7)^{2}$, i.e., $>50 \%$ of the variation was extracted by that factor. On F4, the grouping criterion was $\mid$ loading $\mid \geq 0.50$ (i.e. $25 \%$ of variance extracted); this was done because no samples had loadings $\geq 0.7$ on F4, yet the 5 samples listed in Table 6 all had much higher loadings on F4 than did any of the remaining 74 samples.

The groups are very distinct, and clearly represent day/night and depth influences. F4 includes 5 samples, all of which are $0-25 \mathrm{~m}$ daytime tows. Eight such tows were included in the analysis. Of the 3 which failed to load high on F4, only 1 had high loadings on any other factor (S82 on F1; Table 6). Factor \#3 included 4 daytime samples from deep water (three from 350-600 $\mathrm{m}$, one from 100-350 m). There were seven $100-350 \mathrm{~m}$ and five 350-600 $\mathrm{m}$ daytime samples in the analysis: of the 8 which did not group on F3, only 2 grouped on some other factor (F1; Table 6).

Factor \#2 included 6 samples, all nighttime, from 0-25 and 25-50 $\mathrm{m}$ (Table 6). There were 7 nighttime 0-25 $\mathrm{m}$ samples and 5 night $25-50 \mathrm{~m}$ samples in the analysis (total $=12$ ): none of the 12 grouped on any factor other than F2. Shulenberger (1978) showed that 4 of the 10 most abundant species are much more abundant at $0-25 \mathrm{~m}$ during night than during day (Hyperietta vosseleri, Hyperioides sibaginis, Scina crassicornis, and Paratyphis parrus; species \#24, 26. 60 , and 44 respectively). These 4 species were all very abundant in the six F2 samples.

Factor 1 has the largest number of high loadings and is more complex to interpret than are the other factors. It includes both day and night samples, from many different depth ranges. The 19 samples with high loadings on F1 (Table 6) include only 1 sample from $0-25$ $\mathrm{m}$ : this parallels Shulenberger's (1978) results where all of the most abundant hyperiid species occur primarily in the upper $100 \mathrm{~m}$ both day and night, but where most of those species show no pronounced depth preference over the $25-100 \mathrm{~m}$ range. F1 also includes 7 nighttime samples, from somewhat deeper mean depths than are the 19 daytime samples (Table 6). Upon examination of the abundances of species in samples grouped on F1, it is clear that Primno latreillei (the most abundant species; ID \#56) is the link between these otherwise seemingly disparate F1 subgroups. Shulenberger (1978: Fig. 2) showed that the peak in mean abundance for this species shifted downwards at night: a slight reverse diurnal migration by this abundant species is probably the reason for the grouping of both day and night samples on F1

Q-mode analysis, then, shows a distinct shallow daytime set of samples (F4), a distinct deep daytime set (F3), a distinct shallow nighttime set (F2), and a distinct medium-depth daytime set (F1 daytime samples). The medium-depth nighttime samples by and large failed to group, suggesting a wide-ranging mix of species and/or abundances at these depths during nighttime.

\section{Comparison with Recurrent Group Analysis}

Shulemberger (1979) analyzed the entire data set (83 species, 79 samples) using recurrent group analysis and found 6 groups (Table 7 ). In some respects, recurrent group analysis and the present factor analytic results are very similar. For example, recurrent group \#3 included species 2, 32, and 44: these 3 species also grouped together on 1 factor (however, other species also had high loadings on that same factor: Table 5). Member species of recurrent groups \#4 (Species 53 and 56) and \#5 (Species 25 and 61) were also grouped by factor analysis (Table 5).

The largest recurrent group (\#1; Table 7) included 7 species, which factor analysis placed into 3 separate groups. Because recurrent group analysis works only on presence/absence, it is possible for several speciesspecific patterns of numerical variation to occur simultaneously within a recurrent group. Several species

Table 7. Recurrent group analysis results compared to factor analytic results. Groups: Recurrent group number (Shulenberger, 1979). ID\#: Species identification number (Table 1). F\#: Number of factor on which the species had high loadings

\begin{tabular}{|c|c|c|c|}
\hline & ID \# & $\begin{array}{c}\text { F\# } \\
\text { VARIMAX } \\
\text { (Orthogonal) }\end{array}$ & $\begin{array}{c}\text { F\# } \\
\text { OBLIQUE } \\
\text { (pattern) }\end{array}$ \\
\hline \multirow{7}{*}{ Group I } & 6 & 1 & 3 \\
\hline & 20 & 3 & 2 \\
\hline & 23 & $\cdot$ & $\cdot$ \\
\hline & 24 & 2,3 & 1,2 \\
\hline & 29 & 1 & 3 \\
\hline & 35 & 2 & 1 \\
\hline & 52 & 2 & 1 \\
\hline \multirow{4}{*}{ Group II } & 7 & 1 & 3 \\
\hline & 13 & $\cdot$ & $\cdot$ \\
\hline & 15 & $\cdot$ & - \\
\hline & 26 & 2 & 1 \\
\hline \multirow{3}{*}{ Group III } & 2 & 2 & 1 \\
\hline & 32 & 2 & 1 \\
\hline & 44 & 2 & 1 \\
\hline \multirow{2}{*}{ Group IV } & 53 & 3 & 2 \\
\hline & 56 & 3 & 2 \\
\hline \multirow{2}{*}{ Group V } & 25 & 4 & 5 \\
\hline & 61 & 4 & 5 \\
\hline \multirow{2}{*}{ Group II } & 60 & 5 & 4 \\
\hline & 16 & 1 & 3 \\
\hline
\end{tabular}


may always co-occur, yet fail to show significant covariation. Co-occurring species may respond numerically to entirely different environmental factors, or in different ways to the same factor. This would be particularly true if (a) the apparent co-occurrence is a sampling artifact or (b) if the co-occurrence is real, but is occasioned by physical forces which do not themselves act to change population densities per se (e.g. settling upon density interfaces).

The difference between results of the two techniques may be caused by the failure of recurrent group analysis to utilize much of the information in the data. In recurrent group analysis, only the fact of co-occurrence and numbers of co-occurrences matter, and the latter is compared against a null hypothesis of random cooccurrence assuming each species has occurred randomly in samples. In factor analysis, numerically large co-occurrences could dominate a general pattern of non-co-occurrence, resulting in groupings of species which recurrent group analysis did not group. The converse is also possible. Species might always cooccur yet show no parallels in numerical abundance: then, factor analysis may fail to group them while recurrent group analysis would clearly do so. Any of these may be a possible explanation of the sub-division of the large recurrent group by factor analysis.
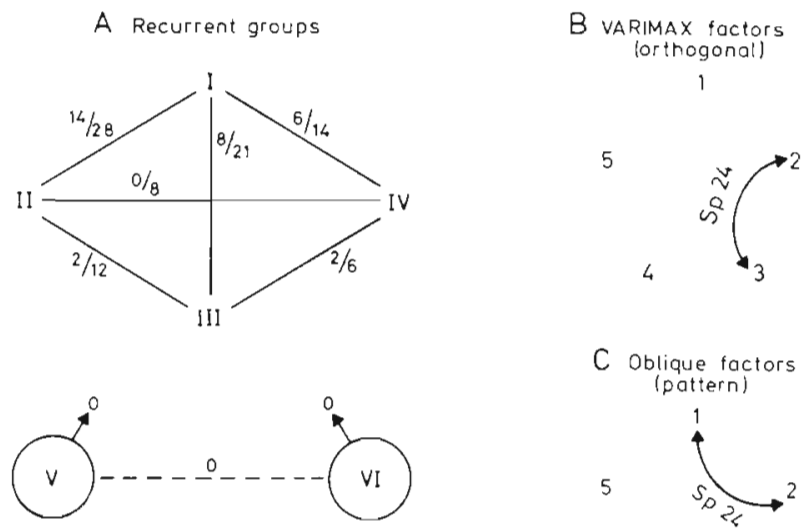

C oblique factors (pattern)

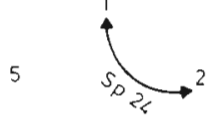

$4 \quad 3$

Fig. 3. Interconnection of groups. (A): Recurrent group analysis. Roman numerals represent group numbers. On lines connecting groups, the denominator of the fraction is the total number of measures of co-occurrence between numbers of the two groups: numerator $=$ number of such measures which were significant (@P 0.01). (B), (C): Orthogonal and oblique groups: only Species 24 had high loadings on as many as two factors

In recurrent group analysis, a species may fail to be a member of a group, yet still have statistically significant frequencies of co-occurrence with some members of that group: such a species may itself be a member of yet another recurrent group. Figure $3 \mathrm{~A}$ shows the pat- tern of interrelationships between recurrent groups: between species of Group I (= 7 spp.) and Group II ( $=$ 4 spp.) there are 28 pairs of species for which co-occurrence indices can be calculated. Fourteen of those were significant (at $p<0.01$; Shulenberger, 1979). In recurrent group analysis only the smallest groups were totally unconnected by significant measures of cooccurrence between members of different groups. By contrast, in both the oblique and orthogonal factor analysis, all groups (large and small) tended to be independent (very little crossover or sharing of species between factors: Fig. 3B, C).

Within the list of factor-analyzed variables are several sets of closely related species: \#6 and 7 (Brachyscelus crusculum and Anchylomera blossevillei) are confamilials, as are \#53 and 56 (Phrosina semilunata and Primno latreiller). Species \#13 and 15 (Eupronoe armata and E. 'sp. A') are congeners, and so are \#29 and 32 (Lestrigonus bengalensis and L. schizogeneios). Within each closely-related set, member species are morphologically very similar (see Shulenberger, 1977. 1978, 1979): for example, Eupronoe armata and E. 'sp. $A^{\prime}$ have only recently been separated. Because of the similarity in body plan within such a set, the animals appear to be likely to have similar lifestyles, and could certainly be in competition with one another if they cooccurred. Recurrent group analysis placed congeners \#20, 23 and 24 (Hyperietta luzoni, stephenseni, and vosseleri) into group \#1, the Eupronoe congeners (\#13 and 15) into group \#2, and confamilials \#53 and 56 into group \#4. This is an apparent contradiction to the competitive exclusion hypothesis, which factor analysis fails to clarify. Factor analysis partially separated the set of three Hyperietta congeners (\#20,23, 24), and usually placed neither of the Eupronoe species (\#13 and 15) into any group. However, the technique confirmed the close parallels of confamilials \#53 and 56 , while also grouping confamilials \#6 and 7 in all analyses. These results suggest that the two descriptive parameters used (presence/absence and abundance) are to some degree independent of one another, particularly within recurrent Group I (Table 7).

\section{Identification of Factors}

The identification of factors with environmental variables is difficult. A priori there is some reason to expect the existence of one pattern per species (the 'one species, one niche' hypothesis). This is not what the R-mode factor analyses detected. Rather, there are only about five major patterns of numerical variation among the 20 most abundant species, suggesting that there are only a few $(\mathrm{N}<<83$; the total hyperiid species list) important processes or underlying forcing 
functions for those patterns. A single factor seems to be dominant in determining the abundance of each species (except Hyperietta vosseleri, \#24; Tables 2, 3). No detailed simultaneous measurements were made of environmental parameters when these samples were taken, hence it is not possible to examine environmental details as candidates for identifications with factors.

It also seemed likely, a priori, that species with similar depth distributions might well covary and should therefore have high loadings upon the same factor. However, the situation is not that simple. The
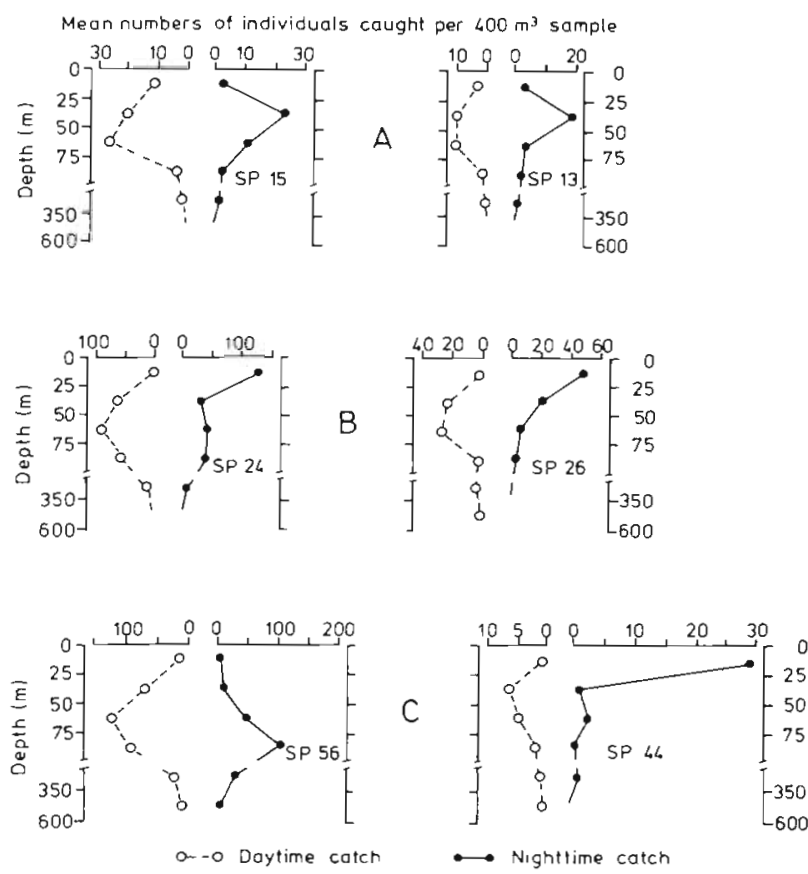

Fig. 4. Depth distributions of species (names given in Table 1). Dots are means of several replicates. Numbers of replicates, $95 \%$ confidence limits, and actual data are all shown in Shulenberger, 1978

Eupronoe congeners (\#13 and 15) had very similar depth and diurnal vertical migration patterns (Fig. $4 \mathrm{a}_{\text {i }}$ see also Shulenberger, 1979), yet failed to have high loadings on any factor (Table 2) and did not group together in factor analysis. They did group together in recurrent group analysis (Table 7). Hyperietta vosseleri (\#24) and Hyperioides sibaginis (\#26), however, also have very similar depth and diurnal vertical migration patterns (Fig. 4b), and were grouped together by factor analysis (Table 2): they failed to co-occur in samples frequently enough to be grouped together by recurrent group analysis (Table 7). H. vosseleri (\#24) and Paratyphis parvus (\#44) have relatively similar day and night distributions (Fig. 4b, c) and grouped together in factor analysis but not in recurrent group analysis. However, $H$. vosseleri (\#24) and Primno latreillei (\#56) have very similar daytime depth distribu- tions, but dissimilar nighttime distributions: factor analysis grouped them together during daytime (Table $5 e)$ but not during the night (Table $5 \mathrm{~d}$ ).

The Q-mode analysis did show groups of similar samples, and those groups seem to reflect day/night, depth distributional, and vertical migration patterns Clearly, depth distributions and diurnal vertical migration patterns are important within the factor analysis, but they are not only underlying process generating variance in the data.

Plots of distribution vs depth have been drawn only for the 10 most abundant species (see Table 1); lowerranked species are sufficiently rare (rank \#11 has only 304 individuals distributed among 79 samples) that the accuracy of depth distributions prepared from their data would be open to serious question. Depth distributions in Figure 4 are based on mean values; Shulenberger (1978) pointed out the danger in using mean value data without measures of variation in such analyses. (Use of means does not permit examination of co-variation.)

One might expect that a few of the most abundant species would in some sense dominate the analysis. There are several ways of examining this: a plot of rank order of abundance against the percentage of total variation (for each species) which is extracted by the analysis is shown in Figure 2 ; the null hypothesis (i.e. no correlation of rank and communality) could be

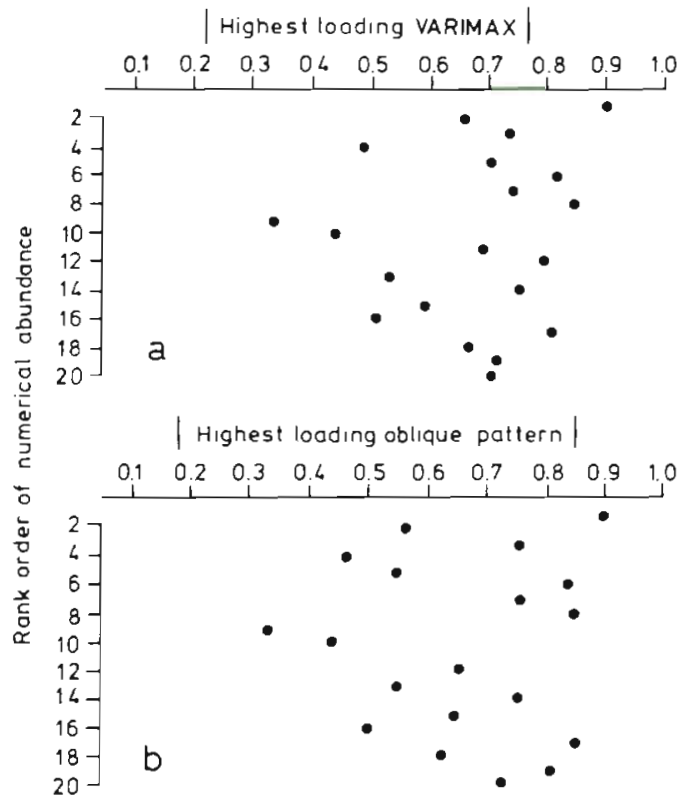

Fig. 5. (A) Species' rank order of numerical abundance vs the highest loading received by that species on any orthogonal (VARIMAX rotation) factor (see Table 1). (B) Species rank order of numerical abundance vs the highest loading received by that species on any oblique (pattern) factor (see Table 1). In both cases the null hypothesis of no correlation was tested using Olmstead and Tukey's 'corner test' for association; $\mathrm{H}_{0}$ accepted, $\mathrm{P}>0.10$, for both cases 
rejected only at $P=0.10$. Figure $5 \mathrm{~A}$ shows there to be no significant relationship between species rank and the maximum loading received by that species on any VARIMAX factor (Olmstead and Tukey's test for association, $P>0.10$ ). Figure 5B likewise shows that there is no significant correlation of species rank with maximum loading on any oblique (pattern) factor (same test, $\mathrm{P}>0.10$ ). If the numerical abundances of $\mathrm{a}$ few of the commonest species dominated the factor results, then there should be negative correlations in Figures 2 and 5 ; only Figure 2 even marginally shows that effect.

Other possible causes for co-variation include aspects of natural history. Recent observations show strong associations of some hyperiid species with species of other major taxa such as salps and ctenophores (Madin and Harbison, 1977; Harbison et al., 1977). Those hyperiid species so reported include some of the North Pacific species plus many congeners of central Pacific species. If, say, 3 species of hyperiids were dependent upon 3 different aspects of the ecology of a particular ctenophore species, the hyperiids might covary numerically depending upon the population density of the ctenophore: the ctenophore might then be the 'factor' accounting for the observed interhyperiid co-variations. However, at present there is no data available on the associations of North Pacific amphipods that can be used to explain any of the observed groupings of species.

Angel and Fasham $(1973,1974,1975)$ factor-analysed data on several major pelagic taxonomic categories (ostracods, chaetognaths, amphipods, euphausiids, and copepods). They found several factors (groups) and related them primarily to depth of tow: the present analyses did not find depth distributions to be an overriding aspect of group composition. They also found, unlike the present analysis, that several species loaded high on 'two or more' factors: the present factor analyses found only two species with high loadings on two factors, and none with such loadings on more than two factors. The species which Angel and Fasham found to load onto several factors had broad depth ranges, and thus overlapped sets of species with more restricted ranges. Since Angel and Fasham (1975) obtained groups while using a more stringent grouping criterion ( | loading $\mid \geq 0.7$ ), ostracod pattern at least may be more pronounced than hyperiid. It is clear, however, that depth distribution is not the central organizing principle in the hyperiid analysis. The different results of this and Angel and Fasham's studies probably reflect (a) the coarser depth strata $(100 \mathrm{~m})$ and greater depth range $(0-2000 \mathrm{~m})$ used by Angel and Fasham, and (b) the fact that their samples were taken over a longer time span in a more advective environment (the Canary Current).
Results of factor analysis and recurrent group analysis are generally parallel, which suggests that patterns of numerical co-variation and co-occurrence in samples are reasonably interactive. Co-occurrence and abundance responses clearly can be different and separable aspects of species' ecologies. Both are of obvious ecological importance; most zooplankton are physically small (a few $\mathrm{mm}$ ) and probably must closely co-occur if they are to interact directly. Recurrent group analysis delineated groups of potentially interacting species, but made no inferences as to the nature (or even existence) of such interactions. The local abundance of a species may be affected by various forces in the environment. Certainly much important information is missing from the present factor analysis: light, nutrient and chlorophyll concentrations, time of day, and finestructure of physical oceanographic parameters should be included in future efforts. Because of known high spatial and temporal variability in those parameters, the use of mean values is contraindicated; those data must be taken concurrently with, and in the same place as, the abundance data.

\section{CONCLUSIONS}

R-mode factor analysis has identified, from the original data on 20 species, 5 sets of co-varying s pecies suggestive of the existence of underlying forcing functions. Although the analysis does not make any inferences as to what those underlying factors might be, they appear to include aspects of taxonomic classification, overall vertical distribution patterns, co-occurrence in samples, overall abundance, and patterns of diurnal vertical migration. None of these appears to dominate the R-mode analyses. Q-mode analysis showed clear patterns of similar samples; those patterns are dominated by time, depth, and vertical migration.

There appear to be many fewer basic organizing forces (factors) underlying structure in the data than there are species (variables) in the analysis. Most species appear to be strongly correlated with only one such factor. Identification of the extracted factors with real-world variables or processes has not yet been accomplished.

The recurrent group and factor analyses combine to suggest that the patterns of numerical abundance of hyperiid species are not very well 'explained' in terms of presences or abundances of other hyperiid species. This suggests that the hyperiid species are not all responding in similar fashion to whatever environmental factors cause numerical variations (hence several factors exist). It also suggests that the forces to which 
various hyperiid species are responding numerically do not, by and large, appear to include other hyperiid species (e.g. probably not predator/prey relationships, etc.) or purely physical forces. These general results appear compatible with observations of tight couplings between some hyperiids and species of other major taxa. Future analyses should not be restricted to only one major taxonomic group, but must include those groups suggested by such observations, as well as including detailed environmental information.

Acknowledgements. Contemplation and writing time were supported by the U.S. Navy Office of Naval Research (Arctic Program, Code 461: contract N00014-78-C-0697). Laboratory work was supported by the Office of Naval Research Ocean Science and Technology Division (Code 480: contract N00014-76-C-0059) and by N.S.F. Grant GA-38009. I am indebted to Dr. M. V. Angel for his constructive criticism. My hyperiid studies originated under and were guided by Dr. J. A. McGowan, of Scripps Institution of Oceanography ${ }_{i}$ he was also responsible for the collection of the plankton samples upon which these analyses are based. I gratefully acknowledge his continued help.

\section{LITERATURE CITED}

Angel, M. V., Fasham, M. J. R. (1973) SOND Cruise (1965) Factor and cluster analyses of the plankton results, a general summary. J. mar. biol. Ass. U. K. 53: 185-231

Angel, M. V., Fasham, M. J. R. (1974). SOND Cruise (1965) Further factor analyses of the plankton data. J. mar. biol. Ass. U. K. 54: 879-894

Angel, M. V., Fasham, M. J. R. (1975). Analysis of the vertical and geographic distribution of the abundant species of planktonic ostracods in the North-East Atlantic. J, mar biol. Ass. U. K. 55: 709-737

Ekman, S. P. (1953). Zoogeography of the sea, Sidgwick and Jackson, London

Fager, E. W. (1957). Determination and analysis of recurrent groups. Ecology 38: 586-595

Fager, E. W., McGowan, J. A. (1963). Zooplankton species groups in the North Pacific. Science, N. Y. 140: 453-460

Hamner, W. M., Madin, L. P., Alldredge, A. L., Gilmer, R. W., Hamner, P. P. (1975). Underwater observations of gelatinous zooplankton: sampling problems, feeding biology, and behavior Limnol. Oceanogr 20: 907-917

Harbison, G. R, Biggs, D. C., Madin, L. P. (1977). The associations of Amphipoda Hyperiidea with gelatinous zooplankton - II. Associations with Cnidaria, Ctenophora, and Radiolaria. Deep Sea Res. 24: 465-488
Harbison, G. R., Madin, L. P., Swanberg, N. R. (1978). On the natural history and distribution of oceanic ctenophores. Deep Sea Res. 25: 233-256

Harman, H. H. (1967). Modern factor analysis (2nd ed. revised), University of Chicago Press, Chicago

Madin, L. P., Harbison, G. R. (1977). The associations of Amphipoda Hyperiidea with gelatinous zooplankton - I. Associations with Salpidae. Deep Sea Res. 24: 449-463

McGowan, J. A. (1971). Oceanic biogeography of the Pacific. In: Funnell, B. M., Reidel, W. R. (eds) The micropaleontology of oceans. Cambridge University Press, Cambridge, pp. 3-74

McGowan, J. A. (1974). The nature of oceanic ecosystems. In: Miller, C. B. (ed.) The biology of the oceanic Pacific Oregon University Press, Oregon, pp. 9-28

McGowan, J. A., Walker, P. W. (in press). Structure in the copepod fraction of the community of the North Pacific central gyre. Ecol. Monogr.

Nie, N. H., Hall, C. H., Jenkins, J. G., Steinbrenner, K., Bent, D. H. (1975). Statistical package for the social sciences (2nd ed.), McGraw-Hill, New York

Reid, J. L., Jr. (1962). On the circulation, phosphate-phosphorus content, and zooplankton volumes in the upper part of the Pacific Ocean. Limnol. Oceanogr. 7: 287-306

Reid, J. L., Brinton, E., Fleminger, A., Venrick, E. L., McGowan, J. A. (1978). Ocean circulation and marine life. In: Charnock, H., Deacon, G. (eds) Advances in Oceanography. Plenum, New York, pp. 65-130

Rottman, M. L. (1978). Ecology of recurrent groups of pteropods, euphausiids, and chaetognaths in the Gulf of Thailand and the South China Sea. Mar. Biol. 48: 63-78

Rummel, R. J. (1970). Applied factor analysis. Northwestem University Press, Evanston, Illinois.

Scripps Institution of Oceanography (1966). A new openingclosing paired zooplankton net. Scripps Institution of Oceanography ref. 66-23: 1-56

Scripps Institution of Oceanography (1974). Data report, physical, chemical, and biological data. CLIMAX I Expedition, 19-28 Sept. 1968. Scripps Institution of Oceanography ref. $74-20$ : $1-41$

Shulenberger, E. (1976). Spatial and associational pattern in the hyperiid amphipod assemblage of the North Pacific central gyre. Ph. D. thesis, Scripps Institution of Oceanography, University of California at San Diego

Shulenberger, E. (1977). Hyperiid amphipods from the zooplankton community of the North Pacific central gyre. Mar. Biol. 42: 375-385

Shulenberger, E. (1978). Vertical distributions, diumal migrations, and sampling problems of hyperiid amphipods in the North Pacific central gyre. Deep Sea Res. 25: 605-623

Shulenberger, E. (1979). Spatial and associational pattern in the hyperiid amphipod assemblage of the North Pacific central gyre. Deep Sea Res. 26A: 293-315

Sokal, R. R., Rohlf, F. J. (1969). Biometry (lst ed.), Freeman, San Francisco 